\title{
Elliptic Double Box and Symbology Beyond Polylogarithms
}

\author{
Alexander Kristensson, Matthias Wilhelm@, and Chi Zhang ๑ \\ Niels Bohr International Academy, Niels Bohr Institute, Copenhagen University, \\ Blegdamsvej 17, 2100 Copenhagen Ø, Denmark
}

(Received 27 July 2021; revised 4 November 2021; accepted 22 November 2021; published 17 December 2021)

\begin{abstract}
We study the elliptic double-box integral, which contributes to generic massless QFTs and is the only contribution to a particular 10-point scattering amplitude in $\mathcal{N}=4$ SYM theory. Based on a Feynman parametrization, we express this integral in terms of elliptic polylogarithms. We then study its symbol, finding a rich structure and remarkable similarity with the nonelliptic case. In particular, the first entry of the symbol is expressible in terms of logarithms of dual-conformal cross ratios, and elliptic letters only occur in the last two entries.
\end{abstract}

DOI: 10.1103/PhysRevLett.127.251603

Introduction.-Understanding of numbers and functions in QFT in general and in $\mathcal{N}=4$ SYM theory in particular has lead to great progress in calculating scattering amplitudes as well as other quantities.

For one-loop quantities, multiple polylogarithms (MPLs) [1-6] suffice, and this continues to be the case in massless theories for higher loop orders for sufficiently low numbers of external particles. MPLs are characterized via their symbol [7], a tensor product-or word-in so-called letters $\log \left(\phi_{\alpha}\right)$, where $\phi_{\alpha}$ are functions of the kinematic variables. These letters encode the singularity and branch-cut structure and their union is known as symbol alphabet. In cases where the $\phi_{\alpha}$ are rational (or can be simultaneously rationalized), the symbol has made it possible to bootstrap the corresponding amplitudes to very high loop orders, i.e., to make an ansatz based on an assumed symbol alphabet [8] and to fix the coefficients via various constraints such as the Steinmann conditions [9-11] and cluster adjacency [12,13]; see, e.g., Refs. [14-19]. For slightly more legs, however, also symbol alphabets with $\phi_{\alpha}$ occur that are not simultaneously rationalizable [20-23].

Beyond MPLs, infinite towers of more complicated functions occur [24-37]. The simplest of these classes of functions involve integrals over elliptic curves; they have recently been increasingly well understood in terms of socalled elliptic multiple polylogarithms (eMPLs) [38-60]. In particular, also a symbol for eMPLs has been defined $[53,61]$; the symbol letters in this case are $\Omega^{(j)}\left(\tilde{\phi}_{\alpha}\right)$, where $\tilde{\phi}_{\alpha}$ are functions of the images of the kinematics when

Published by the American Physical Society under the terms of the Creative Commons Attribution 4.0 International license. Further distribution of this work must maintain attribution to the author(s) and the published article's title, journal citation, and DOI. Funded by SCOAP ${ }^{3}$. mapped to the torus, which is equivalent to the elliptic curve.

In $\mathcal{N}=4$ SYM theory, the first time elliptic functions occur is the 10-point $\mathrm{N}^{3} \mathrm{MHV}$ amplitude at two-loop order. A particular component of it is given in terms of a single Feynman diagram, the elliptic double-box integral [62], depicted in Fig. 1. This integral was found to satisfy a firstorder differential equation relating it to the $6 \mathrm{D}$ hexagon $[63,64]$, as well as a further second-order differential equation [65]. A fourfold rational integral representation - and a onefold polylogarithmic one-were found [66], as well as a sum representation $[67,68]$. So far, however, it has not been possible to express the elliptic double-box integral in terms of eMPLs.

In this Letter, we express the double-box integral in terms of eMPLs and calculate its symbol, finding a rich structure. In particular, we observe that the symbol satisfies the first-entry condition occurring for MPLs [69]: the letters $\Omega^{(j)}\left(\tilde{\phi}_{\alpha}\right)$ in the first entry combine to $\log (u)$, where $u$ is a dual conformal cross ratio. Similarly, the letters in the second entry combine to logs, such that elliptic letters only occur in the last two entries. Moreover, the symbol makes manifest the differential equation relating the elliptic double-box integral to the 6D one-loop hexagon integral.

The linear reducibility problem in the double box and its resolution.-Let us start with the dual conformal Feynman parameter representation of the elliptic double box [66]:
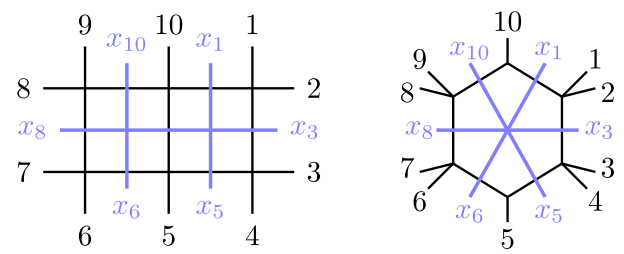

FIG. 1. The elliptic double box and the related 6D hexagon, as well as their dual graphs. 


$$
I_{\mathrm{db}}^{\mathrm{ell}}=\int_{0}^{\infty} d^{4} \vec{\beta} \frac{1}{f_{1} f_{2} f_{3}},
$$

where

$$
\begin{aligned}
& f_{1}=\beta_{4}\left(1+\beta_{1}\right)+\beta_{1}, \\
& f_{2}=1+u_{1} \beta_{4}+v_{1} \beta_{1}+u_{2} \beta_{2}+v_{2} \beta_{3}, \\
& f_{3}=\left(1+u_{3} \beta_{4}\right) \beta_{2}+\left(1+u_{4} \beta_{1}\right) \beta_{3}+\beta_{2} \beta_{3}+u_{3} u_{4} u_{5} f_{1} .
\end{aligned}
$$

The cross ratios are defined by

$$
\begin{aligned}
& u_{1}=x_{1,3 ; 5,8}, \quad u_{2}=x_{3,6 ; 8,10}, \\
& v_{1}=x_{1,8 ; 5,3}, \quad v_{2}=x_{3,10 ; 6,8}, \\
& u_{3}=x_{1,3 ; 5,10}, \quad u_{4}=x_{1,6 ; 5,3}, \quad u_{5}=x_{1,5 ; 6,10},
\end{aligned}
$$

where $x_{a, b ; c, d}=\left(x_{a, b}^{2} x_{c, d}^{2}\right) /\left(x_{a, c}^{2} x_{b, d}^{2}\right)$ with $x_{a, b}=x_{a}-x_{b}$ and dual momenta defined as $x_{a}-x_{a+1}=p_{a}$.

In addition to the manifest dual conformal symmetry, the double-box integral has two reflections symmetries $R_{1}$ and $R_{2}$ along the horizontal and vertical direction in Fig. 1. The action of $R_{1}$ and $R_{2}$ on the cross ratios $\left\{u_{1}, v_{1}, u_{2}, v_{2}, u_{3}\right.$, $\left.u_{4}, u_{5}\right\}$ gives $\left\{v_{1}, u_{1}, v_{2}, u_{2}, u_{4}, u_{3}, u_{5}\right\}$ and $\left\{u_{2}, v_{2}, u_{1}, v_{1}\right.$, $\left.u_{4} u_{2} / v_{1}, u_{3} v_{2} / u_{1}, u_{5}\right\}$, respectively.

As indicated in Ref. [66], three integrations can be performed in terms of polylogarithms, such that the doublebox integral can schematically be expressed as

$$
I_{\mathrm{db}}^{\mathrm{ell}} \sim \int_{0}^{\infty} \frac{d \beta_{1}}{\sqrt{Q\left(\beta_{1}\right)}} H\left(\beta_{1}\right),
$$

where $Q\left(\beta_{1}\right)$ is an irreducible quartic polynomial in $\beta_{1}$ and $H\left(\beta_{1}\right)$ is a pure combination of MPLs of weight three. The obstacle in performing the last integration in terms of elliptic polylogarithms is that the letters of $H\left(\beta_{1}\right)$ involve not only $\sqrt{Q\left(\beta_{1}\right)}$ but also square roots of two quadratic polynomials in $\beta_{1}$. These polynomials share no roots, hence there is no way to rationalize the square roots of the two quadratics without increasing the degree of $Q\left(\beta_{1}\right)$.

To overcome this obstacle, one needs to trace the origin of these additional square roots which are related to the linear reducibility problem of the Feynman parameter integrals [24,57,70,71]. In our case, these square roots of quadratics are introduced in the third integration. More precisely, consider the integral after integrating out $\beta_{3}$ and $\beta_{4}$,

$$
\int_{0}^{\infty} d^{4} \vec{\beta} \frac{1}{f_{1} f_{2} f_{3}}=\int_{0}^{\infty} \frac{d \beta_{1} d \beta_{2}}{\mathcal{P}\left(\beta_{1}, \beta_{2}\right)} \mathcal{G}_{2}\left(\beta_{1}, \beta_{2}\right),
$$

where the polynomial $\mathcal{P}$ has degree 3 and 2 in $\beta_{1}$ and $\beta_{2}$, respectively, and $\mathcal{G}_{2}\left(\beta_{1}, \beta_{2}\right)$ is a pure combination of MPLs of weight two. Three of the letters of $\mathcal{G}_{2}\left(\beta_{1}, \beta_{2}\right)$ are quadratic in $\beta_{1}$ and $\beta_{2}$, while the other letters are linear in $\beta_{1}$ and $\beta_{2}$. It is these three letters that introduce additional square roots in the third integration. To perform the third integration without introducing additional square roots, one needs to make a variable substitution for $\beta_{1}, \beta_{2}$ such that the letters of $\mathcal{G}_{2}$ are linear in one of the new integration variables. A crucial observation here is the following: these three letters, which we denote by $q_{1}, q_{2}$, and $q_{3}$, can be expressed as

$$
\begin{aligned}
& q_{1}=\beta_{1}\left(\beta_{2} u_{2}+\beta_{1} v_{1}\right)+\cdots, \\
& q_{2}=-u_{3}\left(\beta_{2}+\beta_{1} u_{4} u_{5}\right)\left(\beta_{2} u_{2}+\beta_{1} v_{1}\right)+\cdots, \\
& q_{3}=\left(\beta_{2}+\beta_{1} u_{4} u_{5}\right)\left(\beta_{2} u_{2}+\beta_{1} v_{1}\right)+\cdots,
\end{aligned}
$$

where "..." denote terms linear in $\beta_{1}$ and $\beta_{2}$. Then it is natural to introduce the variable substitution

$$
x=\beta_{1} v_{1}+\beta_{2} u_{2}, \quad \tilde{\beta}_{2}=u_{2} \beta_{2} / v_{1},
$$

so that all letters of $\mathcal{G}_{2}$ are linear in $\tilde{\beta}_{2}$ [72]. Now the integration over $\tilde{\beta}_{2}$ gives

$$
I_{\mathrm{db}}^{\mathrm{ell}}=\int_{0}^{\infty} \frac{d x}{y} \mathcal{G}_{3}(x, y),
$$

where

$$
\begin{aligned}
y^{2}= & x^{4}+a_{3} x^{3}+a_{2} x^{2}+a_{1} x+a_{0} \\
= & \left(\frac{v_{1}}{u_{4}}\left[\left(1-u_{4}\right)\left(x+1-v_{2}\right)-u_{1}+u_{3} v_{2}\right]+h_{1}+h_{2}\right)^{2} \\
& -4 h_{1} h_{2},
\end{aligned}
$$

with

$$
\begin{aligned}
& h_{1}=\frac{u_{2} u_{4}}{v_{1}}\left[x^{2}+\left(1-u_{1}+v_{1}\right) x+v_{1}\right], \\
& h_{2}=\left(x+\frac{v_{1}}{u_{4}}\right)\left[\left(1+x-u_{1}\right)\left(\frac{u_{2} u_{4}}{v_{1}}-1\right)+\left(1-u_{3}\right) v_{2}\right] .
\end{aligned}
$$

Here, the coefficients $a_{i}$ are polynomials in the cross ratios, and $\mathcal{G}_{3}$ is a pure combination of MPLs of weight three whose letters are rational functions of $x$ and $y$.

At this stage, there is no obstacle to performing the integration over $x$ and evaluating it in terms of $\mathrm{E}_{4}$ functions which are recursively defined as [50]

$\mathrm{E}_{4}\left(\begin{array}{l}n_{1} \ldots n_{k} \\ c_{1} \ldots c_{k}\end{array} ; x\right)=\int_{0}^{x} d x^{\prime} \psi_{n_{1}}\left(c_{1}, x^{\prime}\right) \mathrm{E}_{4}\left(\begin{array}{l}n_{2} \ldots n_{k} \\ c_{2} \ldots c_{k}\end{array} ; x^{\prime}\right)$,

with $\mathrm{E}_{4}(; x)=1$, where 


$$
\begin{aligned}
& \psi_{0}(0, x)=\frac{1}{y}, \quad \psi_{-1}(\infty, x)=\frac{x}{y}, \\
& \psi_{1}(c, x)=\frac{1}{x-c}, \quad \psi_{-1}(c, x)=\frac{y_{c}}{y(x-c)},
\end{aligned}
$$

with $y_{c}=\left.y\right|_{x=c}$. The definition of $\psi_{n}(c, x)$ for $|n|>1$ can be found in Ref. [50]; the kernels (12) are sufficient for the computation of the double-box integral, though.

We give the final result in terms of $\mathrm{E}_{4}$ functions in the Supplemental Material [73]. Here, we only record the arguments $c_{i}$ of the $\mathrm{E}_{4}$ 's, which make up the set

$$
\begin{aligned}
& \left\{0,-1, \infty,-u_{2},-v_{1},-\frac{v_{1}}{u_{4}},-1+\frac{u_{1}}{u_{3}},-u_{2} u_{4} u_{5},-u_{2}\left(1-u_{4}\right)-v_{1}, \frac{u_{2}\left(u_{3}+u_{4}-1\right)-v_{1}}{1-u_{3}}, \frac{u_{2} u_{3} u_{4} u_{5}-v_{1}}{1-u_{3}},\right. \\
& \frac{u_{2} u_{3} u_{4} u_{5}-v_{1}}{u_{4}\left(1-u_{3} u_{5}\right)}, \frac{u_{2}\left(u_{3} u_{4} u_{5} v_{2}-u_{1}\right)}{u_{3} v_{2}-u_{1}}, \frac{v_{1}\left(u_{3} u_{4} u_{5} v_{2}-u_{1}\right)}{u_{4}\left(u_{1}-u_{3} u_{5} v_{2}\right)}, \frac{u_{4} u_{5}\left[u_{2}\left(u_{4}-1\right)-v_{1}\right]+v_{1}}{u_{4}\left(u_{5}-1\right)}, \frac{u_{1} u_{2}\left(u_{4}-1\right)-v_{1}\left(u_{1}-u_{3} v_{2}\right)}{u_{1}-u_{3} v_{2}}, \\
& z_{1,3,5,8}-1, \bar{z}_{1,3,5,8}-1, z_{1,3,6,8}-1, \bar{z}_{1,3,6,8}-1,-z_{3,5,8,10},-\bar{z}_{3,5,8,10},-z_{3,6,8,10},-\bar{z}_{3,6,8,10}, \\
& \left.\frac{u_{2} u_{3} u_{4} u_{5}-v_{1}+r_{+}}{1-u_{3}}, \frac{u_{2} u_{3} u_{4} u_{5}-v_{1}+r_{-}}{1-u_{3}}\right\},
\end{aligned}
$$

where $z_{a, b, c, d} \bar{z}_{a, b, c, d}=x_{a, b ; c, d},\left(1-z_{a, b, c, d}\right)\left(1-\bar{z}_{a, b, c, d}\right)=$ $x_{d, a ; b, c}$, and

$$
r_{ \pm}=\frac{\mathcal{G}_{45}^{-1} \operatorname{det} \mathcal{G} \pm \sqrt{\operatorname{det} \mathcal{G}^{(45)}} \sqrt{-\operatorname{det} \mathcal{G}}}{2\left(1-u_{5}\right) x_{1,5}^{2} x_{3,10}^{2} x_{1,6}^{2} x_{3,8}^{2} x_{5,10}^{2}} .
$$

Here, we have introduced the Gram matrix $\mathcal{G}=\left(x_{i, j}^{2}\right)$ with $i$ and $j$ running over the set $\{1,3,5,6,8,10\}$, the elements of the inverse of the Gram matrix $\mathcal{G}_{i j}^{-1}=\left(\mathcal{G}^{-1}\right)_{i j}$, as well as the matrix $\mathcal{G}^{(i j)}$ obtained from $\mathcal{G}$ by deleting the $i$ th and $j$ th rows and columns.

From the elliptic curve to the torus: A birational approach.-To define the pureness of the double-box integral and to evaluate its symbol, one needs to express it in terms of iterated integrals on the torus. To this end, we need to find a bijection between the elliptic curve $\mathcal{C}$ and the torus $\mathbb{C} / \Lambda$, where $\Lambda$ is the lattice generated by the periods $\omega_{1}$ and $\omega_{2}$ of the elliptic curve. Instead of using the map provided in Ref. [50], here we adopt another strategy: first we find the standard Weierstrass form $Y^{2}=4 X^{3}-g_{2} X-$ $g_{3}$ birationally equivalent to $\mathcal{C}$ based on its rational point at infinity, then use the standard map $z \mapsto(X, Y)=$ $\left(\wp(z), \wp^{\prime}(z)\right)$ in terms of the Weierstrass $\wp$ function. This gives $z \mapsto(x, y)=\left(\kappa(z), \kappa^{\prime}(z)\right)$, where

$$
\kappa(z)=\frac{6 a_{1}-a_{2} a_{3}+12 a_{3} \wp(z)-24 \wp^{\prime}(z)}{3 a_{3}^{2}-8\left[a_{2}+6 \wp(z)\right]} .
$$

There are several comments in order: (i) the infinity point $(+\infty,+\infty)$ is mapped to a lattice point, (ii) each point $c$ in kinematic space corresponds to two points $\left(c, \pm y_{c}\right)$ on the elliptic curve $\mathcal{C}$ and hence to two images on the torus $\mathbb{C} / \Lambda$, which we denote by $z_{c}^{ \pm}$; these two images satisfy

$$
z_{c}^{+}+z_{c}^{-}=z_{\infty}^{-}+z_{\infty}^{+} \equiv z_{\infty}^{-} \bmod \Lambda,
$$

since the corresponding points $\left(X_{c}^{ \pm}, Y_{c}^{ \pm}\right)$, together with $\left(X_{\infty}^{-}, Y_{\infty}^{-}\right)$, are on the same line. Similarly, one can find that the torus images $z_{c_{i}}^{ \pm}$of the kinematics $c_{i}$ in Eq. (13) satisfy

$$
\begin{aligned}
& \left\{z_{c_{6}}^{+}+z_{c_{7}}^{+}, z_{c_{25}}^{+}+z_{c_{26}}^{+}, z_{c_{6}}^{+}+z_{c_{10}}^{+}+z_{c_{16}}^{+},\right. \\
& \left.\quad z_{c_{10}}^{+}-z_{c_{17}}^{+}+z_{c_{18}}^{+}, z_{c_{16}}^{+}+z_{c_{23}}^{+}-z_{c_{24}}^{+}\right\} \equiv 0 \bmod \Lambda,
\end{aligned}
$$

and similar relations for $z_{c_{i}}^{-}$obtained by Eq. (16), where $c_{i}$ is the $i$ th element in Eq. (13).

In what follows, we will work in the region given by positive momentum-twistor kinematics [74,75], where the four roots of $y^{2}(x)$ come in complex conjugate pairs as shown in Fig. 2. In this case, the torus image $z_{c}^{+}$for any real $c$ is simply given by

$$
z_{c}^{+}=\int_{-\infty}^{c} \frac{d x}{y} .
$$

Hence, $z_{\infty}^{+}$is one period of the torus, and we choose it to be $\omega_{2}$. The image $z_{c}^{-}$can be obtained by Eq. (16) together with $z_{\infty}^{-}=\int_{\gamma_{-}} d x / y$, and the other period is $\omega_{1}=\int_{\gamma_{1}} d x / y$, where the integration contours are defined in Fig. 2.

Now one can introduce iterated integrals on the normalized torus with periods $\left(1, \tau=\omega_{2} / \omega_{1}\right)[50,53]$ :

$\tilde{\Gamma}\left(\begin{array}{l}n_{1} \ldots n_{k} \\ w_{1} \ldots w_{k}\end{array} ; w\right)=\int_{0}^{w} d w^{\prime} g^{\left(n_{1}\right)}\left(w^{\prime}-w_{1}\right) \tilde{\Gamma}\left(\begin{array}{l}n_{2} \ldots n_{k} \\ w_{2} \ldots w_{k}\end{array} ; w^{\prime}\right)$,

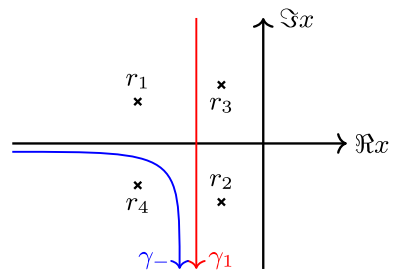

FIG. 2. Four roots of $y^{2}(x)$ in the positive kinematics region and two integration contours. The contour $\gamma_{2}$ which defines $\omega_{2}$ runs along the real axis. 
with $\tilde{\Gamma}(; w)=1$. Such an iterated integral is said to have length $k$ and weight $\sum_{k} n_{k}$. The integration kernels $g^{(n)}(z)$ are generated by the Eisenstein-Kronecker series

$$
\frac{\partial_{z} \theta_{1}(0) \theta_{1}(z+\alpha)}{\theta_{1}(z) \theta_{1}(\alpha)}=\sum_{n \geq 0} \alpha^{n-1} g^{(n)}(z),
$$

where $\theta_{1}(z)=\theta_{1}(z \mid \tau)$ is the odd Jacobi theta function.

With these conventions, it is not hard to find

$$
\begin{gathered}
\psi_{1}(c, x) d x=\left[g^{(1)}\left(w-w_{c}^{+}\right)+g^{(1)}\left(w-w_{c}^{-}\right)\right. \\
\left.-g^{(1)}\left(w-w_{\infty}^{+}\right)-g^{(1)}\left(w-w_{\infty}^{-}\right)\right] d w, \\
\psi_{-1}(c, x) d x=\left[g^{(1)}\left(w-w_{c}^{+}\right)-g^{(1)}\left(w-w_{c}^{-}\right)\right. \\
\left.+g^{(1)}\left(w_{c}^{+}\right)-g^{(1)}\left(w_{c}^{-}\right)\right] d w, \\
\psi_{-1}(\infty, x) d x=\left[g^{(1)}\left(w-w_{\infty}^{-}\right)-g^{(1)}(w)\right. \\
\left.+g^{(1)}\left(w_{\infty}^{-}\right)-\omega_{1} a_{3} / 4\right] d w,
\end{gathered}
$$

as well as $\psi_{0} d x=\omega_{1} d w$, where $w_{c}^{ \pm}$are the normalized torus images $z_{c}^{ \pm} / \omega_{1}$. It is then trivial to express the doublebox integral in terms of $\tilde{\Gamma}$ functions as [76]

$$
I_{\mathrm{db}}^{\mathrm{ell}}=\omega_{1} T_{\mathrm{db}}^{\mathrm{ell}},
$$

where $T_{\mathrm{db}}^{\mathrm{ell}}$ is a pure combination of $\tilde{\Gamma}$ 's of length four and weight three.

Equivalently, the functions $\tilde{\Gamma}$ can be expressed in terms of the functions $\mathcal{E}_{4}$ [54], which are defined in complete analogy to Eq. (11) in terms of kernels $(n \geq 0)$

$$
\begin{aligned}
\Psi_{ \pm n}(c, x) d x= & {\left[g^{(n)}\left(w-w_{c}^{+}\right) \pm g^{(n)}\left(w-w_{c}^{-}\right)\right.} \\
& \left.-\delta_{ \pm n, 1}\left(g^{(1)}\left(w-w_{\infty}^{+}\right)+g^{(1)}\left(w-w_{\infty}^{-}\right)\right)\right] d w .
\end{aligned}
$$

We provide the more compact expression for $T_{\mathrm{db}}^{\mathrm{ell}}$ in terms of $\mathcal{E}_{4}$ 's, as well as code expanding it in terms of $\tilde{\Gamma}$ 's, in the Supplemental Material [73].

Let us close this section by remarking on the shuffle regularization. It will be convenient to introduce $\Omega^{(j)}$, defined via

$$
\begin{aligned}
& \partial_{z} \Omega^{(j)}(z, \tau)=(2 \pi i)^{1-j} g^{(j)}(z, \tau), \\
& \partial_{\tau} \Omega^{(j)}(z, \tau)=j(2 \pi i)^{-j} g^{(j+1)}(z, \tau) .
\end{aligned}
$$

As we shall see in the next section, these $\Omega^{(j)}$ appear as the symbol letters. In contrast to Refs. [40,53], we have included factors of $2 \pi i$ such that all letters have weight 1 and we can find linear relations with rational coefficients among them. By definition, $(2 \pi i)^{1-j} \tilde{\Gamma}\left(\begin{array}{l}j \\ 0\end{array} ; w\right)=\Omega^{(j)}(w)-$ $\Omega^{(j)}(0)$. However, $\boldsymbol{\Omega}^{(1)}(w)$ is singular at $w=0$, and the usual shuffle regularization [53] takes $\tilde{\Gamma}\left({ }_{0}^{1} ; w\right)=\Omega^{(1)}(w)-$ $2 \log \eta(\tau)$ with Dedekind eta function $\eta(\tau)$. Here, to be consistent with the shuffle regularization $G(0 ; x)=\log (x)$ for MPLs which we implicitly used in Eq. (8), we take the shuffle regularization of $\tilde{\Gamma}\left(\begin{array}{l}1 \\ 0\end{array} ; w\right)$ to be

$$
\tilde{\Gamma}\left(\begin{array}{l}
1 \\
0
\end{array} ; w\right)=\Omega^{(1)}(w)-2 \log \eta(\tau)-\log \frac{2 \pi i}{\omega_{1} y_{0}} .
$$

Symbology.-The symbol of $\tilde{\Gamma}$ can be defined recursively via the differential of $\tilde{\Gamma}$ in a similar way as for MPLs [53]. The differential of $\tilde{\Gamma}_{k}^{(n)}$ of weight $n$ and length $k$ schematically takes the form

$$
d \tilde{\Gamma}_{k}^{(n)}=\sum_{i}(2 \pi i)^{j_{i}-1} \tilde{\Gamma}_{k-1}^{\left(n-j_{i}\right)} d \Omega^{\left(j_{i}\right)}\left(y_{i}\right),
$$

where the $\Omega^{(j)}$ are given in Eq. (23) with $\Omega^{(-1)}=-2 \pi i \tau$; the precise formula is given in Ref. [53]. It is easy to see that there would be an overall factor $(2 \pi i)^{n-k}$ if we keep taking the differential recursively. Thus, it is natural to define the symbol for $(2 \pi i)^{k-n} \tilde{\Gamma}_{k}^{(n)}$ rather than $\tilde{\Gamma}_{k}^{(n)}$ as

$\mathcal{S}\left((2 \pi i)^{k-n} \tilde{\Gamma}_{k}^{(n)}\right)=\sum_{i} \mathcal{S}\left((2 \pi i)^{k-n+j_{i}-1} \tilde{\Gamma}_{k-1}^{\left(n-j_{i}\right)}\right) \otimes \Omega^{\left(j_{i}\right)}$.

For the double box, the resulting symbol is of the form

$$
\mathcal{S}\left(T_{\mathrm{db}}^{\mathrm{ell}}\right)=\frac{1}{2 \pi i} \sum_{j} \Omega^{\left(j_{1}\right)}\left(w_{j_{1}}\right) \otimes \cdots \otimes \Omega^{\left(j_{4}\right)}\left(w_{j_{4}}\right),
$$

where $\sum_{i=1}^{4} j_{i}=3$. Naively, there would be $\Omega^{(6)}$ 's at most due to the existence of $\Omega^{(-1)}$, but all $\Omega^{(j>3)}$ 's drop out after using $\Omega^{(j)}(-w)=(-1)^{j+1} \Omega^{(j)}(w)$. At this stage, the symbol has around $10^{6}$ terms.

To make contact with the more familiar kinematic world, we can apply Eq. (21a) to $\int_{a}^{b} \psi_{1}(c, x) d x$ to derive the following identity:

$$
\begin{gathered}
\log \frac{c-a}{c-b}+\sum_{\sigma \in \pm} \Omega^{(1)}\left(w_{c}^{\sigma}-w_{b}^{+}\right)-\Omega^{(1)}\left(w_{c}^{\sigma}-w_{a}^{+}\right) \\
=\sum_{\sigma \in \pm} \Omega^{(1)}\left(w_{\infty}^{\sigma}-w_{b}^{+}\right)-\Omega^{(1)}\left(w_{\infty}^{\sigma}-w_{a}^{+}\right) .
\end{gathered}
$$

Further identities involving elliptic letters can be found using the PSLQ algorithm after numerically evaluating the letters via the sum representations given in the Supplemental Material [73]. For example, we found

$$
\begin{aligned}
& \sum_{i=1}^{6}(-1)^{i+1}\left[\Omega^{(1)}\left(w_{d_{i}}^{-}-w_{\infty}^{+}\right)-\Omega^{(1)}\left(w_{d_{i}}^{-}-w_{0}^{+}\right)\right] \\
& \quad \equiv \log \frac{d_{2}}{d_{3} d_{5}}+\Omega^{(0)}\left(w_{\infty}^{+}-w_{0}^{+}\right) \quad \bmod i \pi
\end{aligned}
$$

with $\quad d_{i} \in\left\{\infty,-v_{1} / u_{4}, \bar{z}_{1,3,5,8}-1, z_{1,3,5,8}-1,-z_{3,6,8,10}\right.$, $\left.-\bar{z}_{3,6,8,10}\right\}$. Moreover, we found complicated identities involving $\Omega^{(2)}$. All these identities turn out to be consequences of Abel's addition theorem and the elliptic Bloch relation [77-79].

Combining (28) and the identities found via the PSLQ algorithm, a dramatic simplification happens: all $\Omega^{(3)}$ 's 
drop out, only logs remain in the first two entries, and the symbol ends up with an expression of around $10^{4}$ terms. Remarkably, the resulting simplified symbol satisfies the same physical first entry conditions found in the MPL case [69], that is the first entries can only be $\log \left(x_{a, b ; c, d}\right)$. Moreover, the symbol follows certain patterns for the first two entries observed in the MPL case in Refs. [23,69,80,81]: the first two entries form the symbols of $\operatorname{Li}_{2}\left(1-x_{a, b ; c, d}\right), \log \left(x_{a, b ; c, d}\right) \log \left(x_{a^{\prime}, b^{\prime} ; c^{\prime}, d^{\prime}}\right)$, or four-mass boxes. In particular, the symbol satisfies the Steinmann conditions $[9,10]$, i.e., discontinuities in partially overlapping channels vanish.

The complete symbol can be organized by its seven elliptic last entries of type $\Omega^{(0)}(w, \tau)=2 \pi i w$ as well as by its behavior under the two reflections $R_{1}, R_{2}$ [73]:

$$
\begin{aligned}
\mathcal{S}\left(T_{\mathrm{db}}^{\mathrm{ell}}\right)= & \mathcal{S}\left(I_{\text {hex }}\right) \otimes\left(w_{c_{25}}^{+}-\frac{w_{\infty}^{+}}{2}\right) \\
& +\mathcal{S}\left(F_{-}\right) \otimes\left(w_{\infty}^{-}-\frac{w_{\infty}^{+}}{2}\right)+\mathcal{S}\left(F_{+}\right) \otimes w_{\infty}^{+} \\
& +\left[\mathcal{S}\left(F_{17}\right) \otimes\left(w_{c_{17}}^{+}-\frac{w_{\infty}^{+}}{2}\right)+\text { reflections }\right],
\end{aligned}
$$

where $I_{\text {hex }}$ is the 6D hexagon integral (normalized to be pure) in Fig. 1 and $F_{+}, F_{-}, F_{17}$ are weight-3 functions whose symbols are known from $\mathcal{S}\left(T_{\mathrm{db}}^{\text {ell }}\right)$ and recorded in the Supplemental Material [73]. In particular, $I_{\text {hex }}, F_{-}$, and $F_{17}$ are polylogarithmic. The symbol can be written in terms of 36 rational letters, 24 algebraic letters (in terms of momentum twistors [74]), and besides the 7 elliptic last entries, elliptic letters only appear at the third entry of $\mathcal{S}\left(F_{+}\right)$and come in only 13 linear independent combinations! (For a list of symbol letters, see the Supplemental Material [73].) The first three terms in Eq. (30) are individually invariant under $R_{1}$ and $R_{2}$. The fourth term generates a 4-orbit, as indicated by the "+ reflections." The precise behavior of the torus images under the reflections is given in the Supplemental Material [73].

Finally, let us remark on the differential equation relating the double box to the $6 \mathrm{D}$ hexagon $[63,64]$. At the level of the symbol it becomes an immediate consequence of Eq. (30) since only $w_{c_{25}}^{+}$in the 7 last entries depends on $u_{5}$ and $\omega_{1} \partial_{u_{5}} w_{c_{25}}^{+}=x_{1,6}^{2} x_{3,8}^{2} x_{5,10}^{2} / \sqrt{-\operatorname{det} \mathcal{G}}$.

Conclusion and outlook.-In this Letter, we have calculated the 10-point two-loop massless double-box integral in terms of eMPLs and calculated its symbol. This integral is the sole contribution to a particular component of the 10-point $\mathrm{N}^{3} \mathrm{MHV}$ superamplitude in planar $\mathcal{N}=4 \mathrm{SYM}$ theory, thus allowing us to draw direct conclusions from our findings for scattering amplitudes.

We find that the symbol of the double-box integral shows a very rich structure. In particular, the first entry of the symbol is drawn from the letters $\log \left(x_{a, b ; c, d}\right)$, where $x_{a, b ; c, d}$ is a dualconformal cross ratio. This means that the double-box integral, despite being elliptic, satisfies exactly the same first-entry conditions that were argued to occur for amplitudes built from nonelliptic polylogarithms. Moreover, the second entry of the symbol contains only letters of log type and satisfies patterns previously observed in the nonelliptic case. The last entry of the symbol is also very restricted, containing only seven possible letters, of elliptic type $\Omega^{(0)}$.

Taking the symbol of our result for the double-box integral, we observed massive cancellations and simplifications, partially due to identities which we first observed numerically via the PSLQ algorithm. As we will elaborate in upcoming work [82], these identities are consequences of Abel's addition theorem and the elliptic Bloch relation [77-79]. It would be interesting to use similar identities to better understand the 13 linearly independent combinations in which the elliptic letters occur in the third entry. Moreover, it would be very interesting to lift this simplified symbol to a simplified function.

The symbol of the double-box integral manifests the differential equation relating it to the 6D one-loop hexagon integral. This suggests that one can bootstrap the symbol via this differential equation, i.e., taking the known symbol of the hexagon, appending the elliptic final letter corresponding to the differential equation, and constructing the remainder of the symbol by imposing integrability [82]. Schematically,

$$
s(\#)=\mathcal{H}(\not{H}) \otimes w_{c_{25}}^{+}+\text {integrability. }
$$

Traintrack integrals [27], which involve integrations over a higher-dimensional Calabi-Yau manifold, similarly satisfy differential equations relating them to $n$-gons $[63,64]$. While the functional space and corresponding symbol is not yet understood in these cases, it seems likely that a bootstrap based on the differential equation will also be possible in these cases.

In the case of MPLs with rational arguments, the symbol alphabets occurring for amplitudes as well as their adjacency conditions can be understood in terms of cluster algebras $[12,13,16]$, and a similar understanding is currently being developed in the case of the Feynman integrals [83-85] and amplitudes including algebraic letters [86-91]. It would be very interesting to use the data we provide in this work to extend the cluster program to the elliptic case. Similarly, it would be interesting to extend the amplitude bootstrap program to the elliptic case.

The double-box integral we considered in this Letter is arguably the simplest elliptic integral contributing to planar $\mathcal{N}=4$ SYM theory, and to massless QFTs in general. We expect that the techniques developed in this Letter can also be applied to more general elliptic integrals, such as the general 12-point double-box integral, corresponding pentabox integrals, and double-pentagon integrals. Combining these integrals with the understanding of prescriptive unitarity and the corresponding leading singularities $[92,93]$ would directly allow us to calculate many further elliptic amplitudes in the massless case. Moreover, also the double-box integral with generic masses is elliptic [94], and should be amenable to the techniques presented here. 
We thank Andrew McLeod and Mark Spradlin for comments on the manuscript, Claude Duhr and Robin Marzucca for communication, as well as Cristian Vergu and Matthias Volk for discussions. C. Z. is grateful to Zhenjie $\mathrm{Li}$ for his instruction on some issues of numeric computations. This work was supported by the research grant 00025445 from Villum Fonden and the ERC starting grant 757978

[1] K.-T. Chen, Iterated path integrals, Bull. Am. Math. Soc. 83, 831 (1977).

[2] A. B. Goncharov, Geometry of configurations, polylogarithms, and motivic cohomology, Adv. Math. 114, 197 (1995).

[3] A. B. Goncharov, Multiple polylogarithms, cyclotomy and modular complexes, Math. Res. Lett. 5, 497 (1998).

[4] E. Remiddi and J. A. M. Vermaseren, Harmonic polylogarithms, Int. J. Mod. Phys. A 15, 725 (2000).

[5] J. M. Borwein, D. M. Bradley, D. J. Broadhurst, and P. Lisonek, Special values of multiple polylogarithms, Trans. Am. Math. Soc. 353, 907 (2001).

[6] S. Moch, P. Uwer, and S. Weinzierl, Nested sums, expansion of transcendental functions and multiscale multiloop integrals, J. Math. Phys. (N.Y.) 43, 3363 (2002).

[7] A. B. Goncharov, M. Spradlin, C. Vergu, and A. Volovich, Classical Polylogarithms for Amplitudes and Wilson Loops, Phys. Rev. Lett. 105, 151605 (2010).

[8] J. Golden, A. B. Goncharov, M. Spradlin, C. Vergu, and A. Volovich, Motivic amplitudes and cluster coordinates, J. High Energy Phys. 01 (2014) 091.

[9] O. Steinmann, Über den Zusammenhang zwischen den Wightmanfunktionen und der retardierten Kommutatoren, Helv. Phys. Acta 33, 257 (1960).

[10] O. Steinmann, Wightman-Funktionen und retardierten Kommutatoren. II, Helv. Phys. Acta 33, 347 (1960).

[11] S. Caron-Huot, L. J. Dixon, F. Dulat, M. von Hippel, A. J. McLeod, and G. Papathanasiou, The cosmic galois group and extended steinmann relations for planar $\mathcal{N}=4 \mathrm{SYM}$ amplitudes, J. High Energy Phys. 09 (2019) 061.

[12] J. Drummond, J. Foster, and Ö. Gürdoğan, Cluster Adjacency Properties of Scattering Amplitudes in $\mathcal{N}=4$ Supersymmetric Yang-Mills Theory, Phys. Rev. Lett. 120, 161601 (2018).

[13] J. Drummond, J. Foster, and Ö. Gürdoğan, Cluster adjacency beyond MHV, J. High Energy Phys. 03 (2019) 086.

[14] S. Caron-Huot, L. J. Dixon, J. M. Drummond, F. Dulat, J. Foster, Ö. Gürdoğan, M. von Hippel, A. J. McLeod, and G. Papathanasiou, The steinmann cluster bootstrap for $\mathcal{N}=4$ super Yang-Mills amplitudes, Proc. Sci. CORFU2019 (2020) 003 [arXiv:2005.06735].

[15] S. Caron-Huot, L. J. Dixon, F. Dulat, M. von Hippel, A. J. McLeod, and G. Papathanasiou, Six-Gluon amplitudes in planar $\mathcal{N}=4$ super-Yang-Mills theory at six and seven loops, J. High Energy Phys. 08 (2019) 016.

[16] J. Drummond, J. Foster, Ö. Gürdoğan, and G. Papathanasiou, Cluster adjacency and the four-loop NMHV heptagon, J. High Energy Phys. 03 (2019) 087.
[17] L. J. Dixon, J. Drummond, T. Harrington, A. J. McLeod, G. Papathanasiou, and M. Spradlin, Heptagons from the Steinmann cluster bootstrap, J. High Energy Phys. 02 (2017) 137.

[18] L. J. Dixon and Y.-T. Liu, Lifting heptagon symbols to functions, J. High Energy Phys. 10 (2020) 031.

[19] L. J. Dixon, A. J. McLeod, and M. Wilhelm, A three-point form factor through five loops, J. High Energy Phys. 04 (2021) 147.

[20] J. L. Bourjaily, A. J. McLeod, C. Vergu, M. Volk, M. Von Hippel, and M. Wilhelm, Rooting out letters: Octagonal symbol alphabets and algebraic number theory, J. High Energy Phys. 02 (2020) 025.

[21] S. He, Z. Li, and C. Zhang, Two-loop octagons, algebraic letters and $\bar{Q}$ equations, Phys. Rev. D 101, 061701(R) (2020).

[22] S. He, Z. Li, and C. Zhang, The symbol and alphabet of twoloop NMHV amplitudes from $\bar{Q}$ equations, J. High Energy Phys. 03 (2021) 278.

[23] S. He, Z. Li, Q. Yang, and C. Zhang, Feynman Integrals and Scattering Amplitudes from Wilson Loops, Phys. Rev. Lett. 126, 231601 (2021).

[24] F. C. S. Brown, On the periods of some Feynman integrals, arXiv:0910.0114.

[25] F. Brown and O. Schnetz, A K3 in $\phi^{4}$, Duke Math. J. 161, 1817 (2012).

[26] J. L. Bourjaily, A. J. McLeod, M. von Hippel, and M. Wilhelm, Bounded Collection of Feynman Integral CalabiYau Geometries, Phys. Rev. Lett. 122, 031601 (2019).

[27] J. L. Bourjaily, Y.-H. He, A. J. McLeod, M. von Hippel, and M. Wilhelm, Traintracks through Calabi-Yau Manifolds: Scattering Amplitudes beyond Elliptic Polylogarithms, Phys. Rev. Lett. 121, 071603 (2018).

[28] D. Festi and D. van Straten, Bhabha Scattering and a special pencil of K3 surfaces, Commun. Num. Theor. Phys. 13, 463 (2019).

[29] J. Broedel, C. Duhr, F. Dulat, R. Marzucca, B. Penante, and L. Tancredi, An analytic solution for the equal-mass banana graph, J. High Energy Phys. 09 (2019) 112.

[30] M. Besier, D. Festi, M. Harrison, and B. Naskrecki, Arithmetic and geometry of a K3 surface emerging from virtual corrections to Drell-Yan scattering, Commun. Num. Theor. Phys. 14, 863 (2020).

[31] J. L. Bourjaily, A. J. McLeod, C. Vergu, M. Volk, M. Von Hippel, and M. Wilhelm, Embedding Feynman integral (Calabi-Yau) geometries in weighted projective space, J. High Energy Phys. 01 (2020) 078.

[32] C. Vergu and M. Volk, Traintrack Calabi-Yaus from twistor geometry, J. High Energy Phys. 07 (2020) 160.

[33] C. F. Doran, A. Y. Novoseltsev, and P. Vanhove, Mirroring towers: Calabi-Yau geometry of the multiloop Feynman sunset integrals (to be published).

[34] D. J. Broadhurst, The master two loop diagram with masses, Z. Phys. C 47, 115 (1990).

[35] L. Adams, E. Chaubey, and S. Weinzierl, Analytic results for the planar double box integral relevant to top-pair production with a closed top loop, J. High Energy Phys. 10 (2018) 206.

[36] L. Adams, E. Chaubey, and S. Weinzierl, Planar Double Box Integral for Top Pair Production with a Closed Top 
Loop to all orders in the Dimensional Regularization Parameter, Phys. Rev. Lett. 121, 142001 (2018).

[37] R. Huang and Y. Zhang, On genera of curves from high-loop generalized unitarity cuts, J. High Energy Phys. 04 (2013) 080 .

[38] S. Laporta and E. Remiddi, Analytic treatment of the two loop equal mass sunrise graph, Nucl. Phys. B704, 349 (2005).

[39] S. Muller-Stach, S. Weinzierl, and R. Zayadeh, From motives to differential equations for loop integrals, Proc. Sci. LL2012 (2012) 005 [arXiv:1209.3714].

[40] F. Brown and A. Levin, Multiple elliptic polylogarithms, arXiv:1110.6917.

[41] S. Bloch and P. Vanhove, The elliptic dilogarithm for the sunset graph, J. Number Theory 148, 328 (2015).

[42] L. Adams, C. Bogner, and S. Weinzierl, The two-loop sunrise graph with arbitrary masses, J. Math. Phys. (N.Y.) 54, 052303 (2013).

[43] L. Adams, C. Bogner, and S. Weinzierl, The two-loop sunrise graph in two space-time dimensions with arbitrary masses in terms of elliptic dilogarithms, J. Math. Phys. (N.Y.) 55, 102301 (2014).

[44] L. Adams, C. Bogner, and S. Weinzierl, The two-loop sunrise integral around four space-time dimensions and generalisations of the Clausen and Glaisher functions towards the elliptic case, J. Math. Phys. (N.Y.) 56, 072303 (2015).

[45] L. Adams, C. Bogner, and S. Weinzierl, The iterated structure of the all-order result for the two-loop sunrise integral, J. Math. Phys. (N.Y.) 57, 032304 (2016).

[46] L. Adams, C. Bogner, A. Schweitzer, and S. Weinzierl, The kite integral to all orders in terms of elliptic polylogarithms, J. Math. Phys. (N.Y.) 57, 122302 (2016).

[47] L. Adams and S. Weinzierl, Feynman integrals and iterated integrals of modular forms, Commun. Num. Theor. Phys. 12, 193 (2018).

[48] L. Adams, E. Chaubey, and S. Weinzierl, Simplifying Differential Equations for Multiscale Feynman Integrals beyond Multiple Polylogarithms, Phys. Rev. Lett. 118, 141602 (2017).

[49] C. Bogner, A. Schweitzer, and S. Weinzierl, Analytic continuation and numerical evaluation of the kite integral and the equal mass sunrise integral, Nucl. Phys. B922, 528 (2017).

[50] J. Broedel, C. Duhr, F. Dulat, and L. Tancredi, Elliptic polylogarithms and iterated integrals on elliptic curves. Part I: General formalism, J. High Energy Phys. 05 (2018) 093.

[51] J. Broedel, C. Duhr, F. Dulat, and L. Tancredi, Elliptic polylogarithms and iterated integrals on elliptic curves II: An application to the sunrise integral, Phys. Rev. D 97, 116009 (2018).

[52] L. Adams and S. Weinzierl, The $\varepsilon$-form of the differential equations for Feynman integrals in the elliptic case, Phys. Lett. B 781, 270 (2018).

[53] J. Broedel, C. Duhr, F. Dulat, B. Penante, and L. Tancredi, Elliptic symbol calculus: From elliptic polylogarithms to iterated integrals of Eisenstein series, J. High Energy Phys. 08 (2018) 014.

[54] J. Broedel, C. Duhr, F. Dulat, B. Penante, and L. Tancredi, Elliptic Feynman integrals and pure functions, J. High Energy Phys. 01 (2019) 023.
[55] I. Hönemann, K. Tempest, and S. Weinzierl, Electron selfenergy in QED at two loops revisited, Phys. Rev. D 98, 113008 (2018).

[56] C. Bogner, S. Müller-Stach, and S. Weinzierl, The unequal mass sunrise integral expressed through iterated integrals on $\overline{\mathcal{M}}_{1,3}$, Nucl. Phys. B954, 114991 (2020).

[57] J. Broedel, C. Duhr, F. Dulat, B. Penante, and L. Tancredi, Elliptic polylogarithms and Feynman parameter integrals, J. High Energy Phys. 05 (2019) 120.

[58] C. Duhr and L. Tancredi, Algorithms and tools for iterated Eisenstein integrals, J. High Energy Phys. 02 (2020) 105.

[59] M. Walden and S. Weinzierl, Numerical evaluation of iterated integrals related to elliptic Feynman integrals, Comput. Phys. Commun. 265, 108020 (2021).

[60] S. Weinzierl, Modular transformations of elliptic Feynman integrals, Nucl. Phys. B964, 115309 (2021).

[61] F. Brown, Notes on motivic periods, arXiv:1512.06410.

[62] S. Caron-Huot and K. J. Larsen, Uniqueness of twoloop master contours, J. High Energy Phys. 10 (2012) 026.

[63] M. F. Paulos, M. Spradlin, and A. Volovich, Mellin amplitudes for dual conformal integrals, J. High Energy Phys. 08 (2012) 072.

[64] D. Nandan, M. F. Paulos, M. Spradlin, and A. Volovich, Star integrals, convolutions and simplices, J. High Energy Phys. 05 (2013) 105.

[65] D. Chicherin and E. Sokatchev, Conformal anomaly of generalized form factors and finite loop integrals, J. High Energy Phys. 04 (2018) 082.

[66] J. L. Bourjaily, A. J. McLeod, M. Spradlin, M. von Hippel, and M. Wilhelm, Elliptic Double-Box Integrals: Massless Scattering Amplitudes beyond Polylogarithms, Phys. Rev. Lett. 120, 121603 (2018).

[67] F. Loebbert, D. Müller, and H. Münkler, Yangian bootstrap for conformal Feynman integrals, Phys. Rev. D 101, 066006 (2020).

[68] B. Ananthanarayan, S. Banik, S. Friot, and S. Ghosh, Double box and hexagon conformal Feynman integrals, Phys. Rev. D 102, 091901(R) (2020).

[69] D. Gaiotto, J. Maldacena, A. Sever, and P. Vieira, Pulling the straps of polygons, J. High Energy Phys. 12 (2011) 011.

[70] E. Panzer, On hyperlogarithms and Feynman integrals with divergences and many scales, J. High Energy Phys. 03 (2014) 071.

[71] J. L. Bourjaily, Y.-H. He, A. J. McLeod, M. Spradlin, C. Vergu, M. Volk, M. von Hippel, and M. Wilhelm, Direct integration for multi-leg amplitudes: Tips, tricks, and when they fail, in Antidifferentiation and the Calculation of Feynman Amplitudes (Springer International Publishing, Cham, 2021), 10.1007/978-3-030-80219-6_5.

[72] The rescaling $\tilde{\beta}_{2}=u_{2} \beta_{2} / v_{1}$ is not essential for linear reducibility but is chosen to set the coefficient of $x^{4}$ in Eq. (9) to 1.

[73] See Supplemental Material at http://link.aps.org/ supplemental/10.1103/PhysRevLett.127.251603 for Mathematica-readable expressions for the function result and symbol result of the double box integral, and supp.pdf for reflection symmetries and the symbol alphabet of the double box. 
[74] A. Hodges, Eliminating spurious poles from gauge-theoretic amplitudes, J. High Energy Phys. 05 (2013) 135.

[75] N. Arkani-Hamed and J. Trnka, The amplituhedron, J. High Energy Phys. 10 (2014) 030.

[76] Without expressing the double box in terms of eMPLs, it was previously argued that it is pure in Ref. [54].

[77] Don Zagier and Herbert Gangl, Classical and elliptic polylogarithms and special values of 1-series, in The Arithmetic and Geometry of Algebraic Cycles, edited by B. Brent Gordon, James D. Lewis, Stefan Müller-Stach, Shuji Saito, and Noriko Yui (Springer Netherlands, Dordrecht, 2000), pp. 561-615.

[78] Spencer J. Bloch, Higher Regulators, Algebraic K-Theory, and Zeta Functions of Elliptic Curves (American Mathematical Society, Providence, 2011), Vol. 11.

[79] Johannes Broedel and André Kaderli, Functional relations for elliptic polylogarithms, J. Phys. A 53, 245201 (2020).

[80] S. Caron-Huot, Superconformal symmetry and two-loop amplitudes in planar $\mathcal{N}=4$ super Yang-Mills, J. High Energy Phys. 12 (2011) 066.

[81] T. Dennen, M. Spradlin, and A. Volovich, Landau singularities and symbology: One- and two-loop MHV amplitudes in SYM theory, J. High Energy Phys. 03 (2016) 069.

[82] M. Wilhelm and C. Zhang (to be published).

[83] D. Chicherin, J. M. Henn, and G. Papathanasiou, Cluster Algebras for Feynman Integrals, Phys. Rev. Lett. 126, 091603 (2021).
[84] S. He, Z. Li, and Q. Yang, Notes on cluster algebras and some all-loop Feynman integrals, J. High Energy Phys. 06 (2021) 119.

[85] S. He, Z. Li, and Q. Yang, Truncated cluster algebras and Feynman integrals with algebraic letters, arXiv:2106.09314.

[86] J. Drummond, J. Foster, Ö. Gürdogan, and C. Kalousios, Algebraic singularities of scattering amplitudes from tropical geometry, J. High Energy Phys. 04 (2021) 002.

[87] N. Arkani-Hamed, T. Lam, and M. Spradlin, Nonperturbative geometries for planar $\mathcal{N}=4$ SYM amplitudes, J. High Energy Phys. 03 (2021) 065.

[88] N. Henke and G. Papathanasiou, How tropical are sevenand eight-particle amplitudes?, J. High Energy Phys. 08 (2020) 005.

[89] A. Herderschee, Algebraic branch points at all loop orders from positive kinematics and wall crossing, J. High Energy Phys. 07 (2021) 049.

[90] N. Henke and G. Papathanasiou, Singularities of eight- and nine-particle amplitudes from cluster algebras and tropical geometry, J. High Energy Phys. 10 (2021) 007.

[91] L. Ren, M. Spradlin, and A. Volovich, Symbol alphabets from tensor diagrams, arXiv:2106.01405.

[92] J. L. Bourjaily, N. Kalyanapuram, C. Langer, K. Patatoukos, and M. Spradlin, Elliptic, Yangian-Invariant "Leading Singularity", Phys. Rev. Lett. 126, 201601 (2021).

[93] J. L. Bourjaily, N. Kalyanapuram, C. Langer, and K. Patatoukos, Prescriptive unitarity with elliptic leading singularities, arXiv:2102.02210.

[94] S. Bloch, Double box motive, SIGMA 17, 048 (2021). 